\title{
Forwarding Repeated Game for End-to-End QoS Support in Fiber-Wireless Access Networks
}

\author{
J. Coimbra*, G. Schütz ${ }^{\dagger}$, N. Correia* \\ ${ }^{*}$ Center for Electronic, Optoelectronic and Telecommunications (CEOT), Faculty of Science and Technology \\ University of Algarve, 8005-139 Faro, Portugal, Email: \{jcoimbra,ncorreia\}@ualg.pt \\ †Institute of Engineering, University of Algarve, 8005-139 Faro, Portugal, Email: gschutz@ualg.pt
}

\begin{abstract}
Fiber-wireless (FiWi) access mesh networks have been proposed as flexible and cost-effective solutions for future access networks. However, for FiWi access mesh networks to provide end-to-end QoS guarantees to its customers, efficient QoS routing schemes and scheduling policies become necessary. Here we provide a model, using repeated game theory, that creates ground for scheduling policies to be implemented. That is, our framework provides insights into how much bandwidth a wireless router needs to share, for foreign traffic forwarding, so that services of local users and services of foreign users can all receive bandwidth in a balanced way. Some strategies were tested, under the developed model, to look for equilibrium states. The equilibrium strategies provide the means for QoS guarantees in FiWi access mesh networks, meaning that they can be used as a basis for scheduling policies.
\end{abstract}

\section{INTRODUCTION}

Optical and wireless communications were initially developed with different objectives [1]. Optical communications were developed for high bandwidth and long distance communications, whereas wireless communications were developed for short distance communications with low bandwidth requirements. However, the rise of bandwidth intensive applications, the way people now communicate, and the advances that have been made in wireless technologies, which allow for much higher bandwidths, are accelerating research in hybrid fiber-wireless (FiWi) access networks. The driving forces for such research are that it may be too expensive and geographically difficult to install fiber to every home, and having a wireless network from the central office (CO) to every home may be technically impossible due to limited spectrum. Therefore, having fiber as far as possible from the $\mathrm{CO}$ to the end users and then wireless at the front end can provide a flexible and cost-effective solution. Besides flexibility, such approach provides higher bandwidth than digital subscriber lines (DSL) or cable modem technologies [2]. There are already proposals for FiWi access mesh networks [3], [4].

In most of the FiWi access network proposals, the wireless front end is composed of wireless routers, which accept connections from users, and wireless gateway routers that are connected to optical network units (ONUs) to send/receive traffic to/from the optical link. For wireless routers to successfully communicate with gateways, packets may need to be forwarded by intermediate wireless routers. Hence, wireless routers have to forward traffic from other routers besides their own. The question is how to provide resources, at each router, in order to meet the requirements of local and foreign communications. Moreover, how to meet that goal throughout the wireless section in an efficient manner. Undoubtedly, two major building blocks are the establishment of efficient QoS routing schemes and scheduling policies. Here we provide a model, using repeated game theory, that creates ground for scheduling policies to be implemented. That is, our framework provides insights into how much bandwidth a wireless router needs to share, for foreign traffic forwarding, so that services of local and foreign users can all receive bandwidth in a balanced way, creating ground for QoS guarantees.

There is already some work using game theory on wireless meshes [5], [6], [7], [8]. However, traditional wireless mesh networks work differently from FiWi access mesh networks. At the former, traffic can be sent towards any node and can flow through any intermediate nodes in the network. A node at the latter only sends/receives traffic to/from the Internet through gateways. As far as we know, repeated game theory has not been previously applied to FiWi networks.

In this paper we address the problem of equilibrium in $\mathrm{FiWi}$ access mesh networks. That is, the possibility of a balance between the shared bandwidth and the bandwidth for the router itself, called here self bandwidth. If there is a good balance in every router, then end-to-end QoS guarantees can be implemented because the performance of the FiWi access mesh network is more predictable. With that in mind, we have created a repeated game theory model, and searched for strategies to be implemented at routers and gateways that enable good bandwidth balancing at the access wireless section. This model may serve as a basis for the development of QoS scheduling policies.

The rest of this paper is organized as follows. Section II describes the FiWi access mesh network model. Section III discusses the proposed repeated game model while introducing concepts from repeated game theory. Section IV discusses simulations and strategies used. Section V analyses the results, and Section VI ends the paper with conclusions.

\section{NETWORK MODEL}

In a FiWi access mesh network, the interface with the user is made using a multi-hop wireless mesh network similar to the one in Fig. 1, the San Francisco Network (SFNet) taken from [4]. For packets to travel from the user to the gateway, 


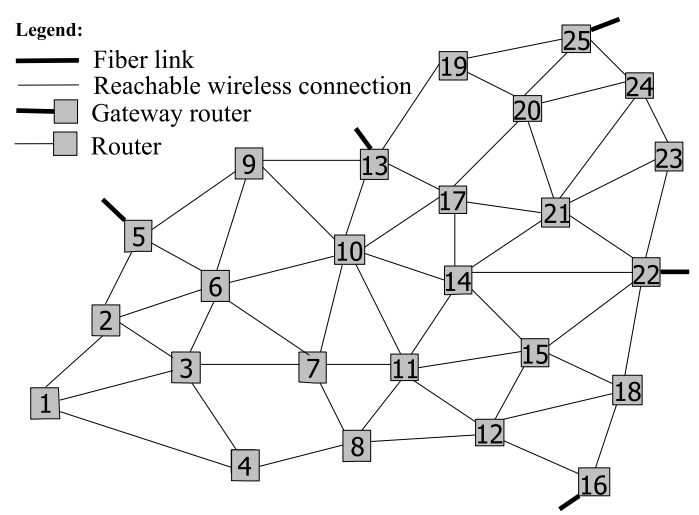

Fig. 1. San Francisco Network (SFNet)

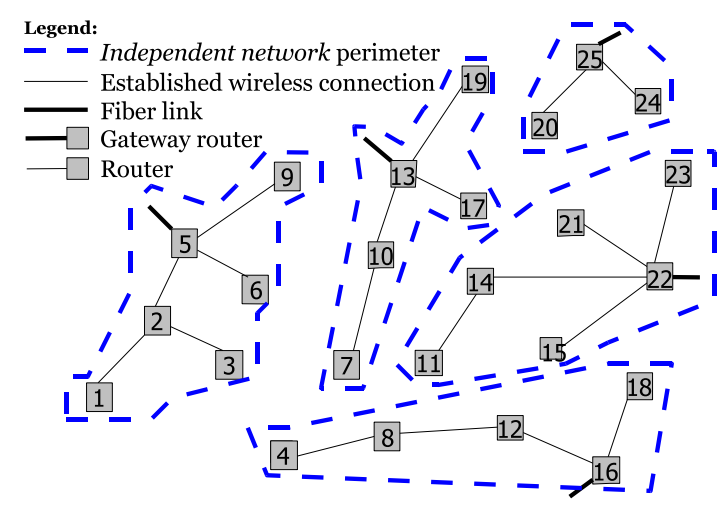

Fig. 2. Independent networks created by the shortest paths calculation.

some routers will have to forward traffic. In this article we assume that traffic flows according to the shortest path.

For a FiWi access mesh network $\mathcal{N}$, with $R$ routers and $G$ gateway routers, let us consider only the links that belong to the shortest path between each router and its nearest gateway router, so that a disconnected network composed of $G$ smaller connected subnetworks, $\mathcal{N}_{1}, \mathcal{N}_{2}, \ldots, \mathcal{N}_{G}$, is obtained, as shown in Fig. 2. In this article these smaller connected networks are called independent networks, having tree structures. Nodes from lower levels in the tree structure depend solely on nodes at higher levels to send and receive traffic to and from the Internet. The model developed in this article is applied to each independent network in a FiWi access mesh network.

Each independent network, $\mathcal{N}_{i}, i=1,2, \ldots, G$, has a gateway, denoted by $g_{i}$, and $n_{i}$ routers, $r_{1}, r_{2}, \ldots, r_{n_{i}}$. Clearly, $\sum_{i=1}^{G} n_{i}=R$. For simplicity, we denote the set of routers in an independent network $\mathcal{N}_{i}$ by $\mathcal{R}_{i}$, and the gateway by $g_{i}$. Every router $r_{j} \in \mathcal{R}_{i}$, for $j=1, \ldots, n_{i}$ and $i=1, \ldots, G$, has a set of users connected to it that together receive downstream traffic, $D_{r_{j}}(t)$, and generate upstream traffic, $U_{r_{j}}(t)$, throughout time $t$.

Every router that is not a leaf in the tree structures (see Fig. 2) has to forward some traffic from foreign users so that the network can work properly. In this article, the routers that are not leafs in the tree structure are called forwarding routers, and the routers that are leafs are called non forwarding routers. The set of forwarding routers from an independent network $\mathcal{N}_{i}, \forall i \in\{1, \ldots, G\}$, is denoted by $\mathcal{F}_{i}$, containing $F_{i}$ forwarding routers, $f_{1}, f_{2}, \ldots, f_{F_{i}}$. Note that $\mathcal{F}_{i} \subset \mathcal{R}_{i}$. Each forwarding router $f_{j}, \forall j \in\left\{1, \ldots, F_{i}\right\}, \forall i \in\{1, \ldots, G\}$, forwards traffic from foreign users according to a variable called cooperation level, $\lambda_{f_{j}}(t) \in[0,1]$, that represents the fraction of forwarded traffic belonging to other wireless routers. The value 0 and 1 represent full defection and full cooperation, respectively. A forwarding router has the freedom to change the cooperation level along time $t$. In this paper, we use the term router to mean either a forwarding router or a non forwarding router, that is not a gateway. We also use the term node to either refer to a router or a gateway.

For a clearer discussion of the model, we assume that gateways do not have users connected to them. However, the model can easily be extended to consider local traffic if the gateway routers are assumed to be composed of two connected parts: an ordinary forwarding router and a gateway router. A gateway $g_{i}, \forall i \in\{1, \ldots, G\}$, only forwards traffic from routers in the same independent network $\mathcal{N}_{i}$. Every gateway $g_{i}$, has a vector $\mu^{g_{i}}(t)$ including the cooperation level with every router $r_{j}$, denoted by $\mu_{r_{j}}^{g_{i}}(t), \forall j \in\left\{1, \ldots, n_{i}\right\}$. That is, $\mu_{r_{j}}^{g_{i}}(t)$ represents the cooperation level applied by the gateway $g_{i}$ to router $r_{j}$, throughout time $t$, and their sum must equal 1 :

$$
\sum_{j=1}^{n_{i}} \mu_{r_{j}}^{g_{i}}(t)=1, \forall i \in\{1, \ldots, G\} .
$$

\section{RePeAted GAMe Model}

\section{A. Notation}

Our repeated game model is inspired by the forwarder's dilemma game [6]. Before getting into the details of the model, the following notation must be introduced:

$G \quad$ Total number of gateways in the FiWi access mesh network. It is also the total number of independent networks since each includes a single gateway.

$R \quad$ Total number of routers in the FiWi access mesh network, except gateway routers.

$\mathcal{N}_{i} \quad$ Independent network that emerges when the shortest path routing is applied.

$\mathcal{R}_{i} \quad$ Set of routers in the independent network $\mathcal{N}_{i}$. This set does not include the gateway of $\mathcal{N}_{i}$.

$\mathcal{F}_{i} \quad$ Set of forwarding routers in the independent network $\mathcal{N}_{i}$.

$g_{i} \quad$ Gateway router in the independent network $\mathcal{N}_{i}$.

$\lambda_{f_{j}}(t)$ Cooperation level of a forwarding router $f_{j}, \forall j \in$ $\left\{1, \ldots, F_{i}\right\}, \forall i \in\{1, \ldots, G\}$, throughout time $t$.

$\mu^{g_{i}}(t)$ Cooperation level vector of gateway $g_{i}, \forall i \in$ $\{1, \ldots, G\}$, throughout time $t$.

$\mu_{r_{j}}^{g_{i}}(t)$ Cooperation level of gateway $g_{i}$ with router $r_{j}, \forall j \in$ $\left\{1, \ldots, n_{i}\right\}, \forall i \in\{1, \ldots, G\}$, throughout time $t$.

$D_{r_{j}}(t)$ Downstream traffic destined to router $r_{j}, \forall j \in$ $\left\{1, \ldots, n_{i}\right\}, \forall i \in\{1, \ldots, G\}$, throughout time $t$.

$U_{r_{j}}(t)$ Upstream traffic from router $r_{j}, \forall j \in$ $\left\{1, \ldots, n_{i}\right\}, \forall i \in\{1, \ldots, G\}$, throughout time $t$. 
$\Gamma_{k}^{+} \quad$ Set that contains all the descendants of node $k$ in its independent network.

$\Gamma_{k}^{-} \quad$ Set that contains all the ancestors of node $k$ in its independent network.

\section{B. Stage Game}

Repeated games are composed of many stages, and in each stage there is a game called stage game. The time $t$, used throughout the article, does not refer to continuous time but rather to stages. The model for the stage game will be explained next.

Let us consider the independent network of gateway 5 in Fig. 2. Node 1 has $D_{1}(t)$ of downstream traffic destined to it. However, the gateway only forwards a fraction, $\mu_{1}^{5}(t)$, of the traffic $D_{1}(t)$ and router 2 only forwards a fraction, $\lambda_{2}(t)$, of the traffic $D_{1}(t) \mu_{1}^{5}(t)$. So, router 1 receives $D_{1}(t) \mu_{1}^{5}(t) \lambda_{2}(t)$ of downstream traffic. Generalizing, the received downstream traffic for any router $r_{j} \in \mathcal{R}_{i}, \forall j \in$ $\left\{1, \ldots, n_{i}\right\}, \forall i \in\{1, \ldots, G\}$, can be calculated using ${ }^{1}$ :

$$
d_{r_{j}}(t)=D_{r_{j}}(t) \mu_{r_{j}}^{g_{i}}(t) \prod_{k \in\left(\Gamma_{r_{j}}^{-} \backslash g_{i}\right)} \lambda_{k}(t) .
$$

The same happens for upstream. Considering router 1, only $U_{1}(t) \lambda_{2}(t) \mu_{1}^{5}(t)$ of traffic from router 1 arrives the optical section. To calculate the upstream traffic, for any router $r_{j} \in$ $\mathcal{R}_{i}, \forall j \in\left\{1, \ldots, n_{i}\right\}, \forall i \in\{1, \ldots, G\}$, we use:

$$
u_{r_{j}}(t)=U_{r_{j}}(t) \mu_{r_{j}}^{g_{i}}(t) \prod_{k \in\left(\Gamma_{r_{j}}^{-} \backslash g_{i}\right)} \lambda_{k}(t) .
$$

The total traffic (downstream and upstream) belonging to the users of a router $r_{j}$ successfully received and transmitted, at stage $t$, will be:

$$
\begin{aligned}
\tau_{r_{j}}(t)= & d_{r_{j}}(t)+u_{r_{j}}(t), \\
& , \forall j \in\left\{1, \ldots, n_{i}\right\}, \forall i \in\{1, \ldots, G\} .
\end{aligned}
$$

Forwarding routers will have to forward traffic besides their own, as already explained in Section II. Such forwarded traffic can be calculated by:

$$
\begin{aligned}
\theta_{f_{j}}(t)= & \alpha_{f_{j}}(t) \lambda_{f_{j}}(t), \\
& , \forall j \in\left\{1, \ldots, F_{i}\right\}, \forall i \in\{1, \ldots, G\},
\end{aligned}
$$

where $\alpha_{f_{j}}$ is the sum of all the traffic that router $f_{j}$ receives/sends from/to other routers:

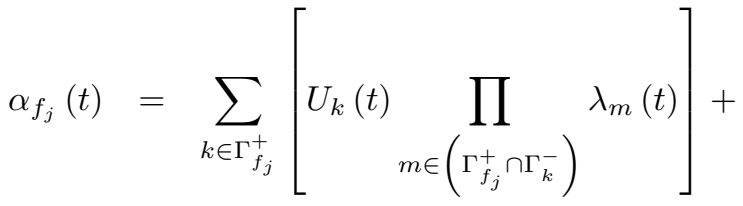

$$
\begin{aligned}
& +\sum_{k \in \Gamma_{f_{j}}^{+}}\left[D_{k}(t) \mu_{k}^{g_{i}}(t) \prod_{m \in\left(\Gamma_{f_{j}}^{-} \cap \Gamma_{g_{i}}^{+}\right)} \lambda_{m}(t)\right] \text {. }
\end{aligned}
$$

\footnotetext{
${ }^{1}$ Note that the product of an empty set equals one.
}

Concerning the gateway, the forwarded traffic can be calculated using the following expression:

$$
\begin{aligned}
\theta_{g_{i}}(t)= & \sum_{k \in \Gamma_{g_{i}}^{+}}\left[D_{k}(t) \mu_{k}^{g_{i}}(t)\right]+ \\
+ & \sum_{\substack{k \in \Gamma_{g_{i}}^{+}\\
}}\left[U_{k}(t) \mu_{k}^{g_{i}}(t) \prod_{m \in\left(\Gamma_{g_{i}}^{+} \cap \Gamma_{k}^{-}\right)} \lambda_{m}(t)\right], \\
& , \forall i \in\{1, \ldots, G\} .
\end{aligned}
$$

To measure the interests of players, stage payoff functions are used. We have different stage payoff functions for forwarding and gateway routers because of their different objectives. The stage payoff of forwarding routers, $v_{f_{j}}(t)$, is calculated by the sum of two functions. The first is a gain function $\rho_{f_{j}}\left(\tau_{f_{j}}(t)\right), \forall j \in\left\{1, \ldots, F_{i}\right\}, \forall i \in\{1, \ldots, G\}$, dependent on the traffic successfully sent and received. The second is a negative cost function $c\left(\theta_{f_{j}}(t)\right), \forall j \in\left\{1, \ldots, F_{i}\right\}, \forall i \in$ $\{1, \ldots, G\}$, that depends on the traffic forwarded to other routers. Thus the stage payoff of a forwarding router will be:

$$
\begin{aligned}
v_{f_{j}}(t)= & \rho_{f_{j}}\left(\tau_{f_{j}}(t)\right)+c\left(\theta_{f_{j}}(t)\right), \\
& , \forall j \in\left\{1, \ldots, F_{i}\right\}, \forall i \in\{1, \ldots, G\} .
\end{aligned}
$$

Therefore, in order to have a high payoff, it is in the interest of the forwarding routers to forward a low amount of foreign traffic, and send a high amount of local traffic.

For the gateway, whose objective is to forward as much traffic as possible, the stage payoff is calculated using just a gain function $\rho_{g_{i}}\left(\theta_{g_{i}}(t)\right), \forall i \in\{1, \ldots, G\}$, that depends on the total amount of traffic the gateway forwards:

$$
v_{g_{i}}(t)=\rho_{g_{i}}\left(\theta_{g_{i}}(t)\right), \forall i \in\{1, \ldots, G\} .
$$

Note that gateways have a higher payoff if they forward more traffic. Therefore, it is in the interest of the gateway that forwarding routers forward a high amount of traffic.

Forwarding routers and gateways are the only nodes that can make decisions about the game since they are the ones that can decide how much traffic to forward. Hence, the forwarding routers and the gateways are the only players in the game.

\section{Repeated Game}

A repeated game consists in a number of repetitions of some stage game. In every stage, players decide the actions to take based on the stage payoff they get. A player can decide for the action that gives him the highest payoff. However, that action can cause loss to other players in the game, and these other players can, in future stages, punish that player. Therefore, in a repeated game, the players have to worry about present and future stage payoffs when deciding which action to take [9]. A forwarding router might choose to defect in order to have a higher payoff, however, the gateway can punish the defecting forwarding router in future stages, leading to a lower future stage payoff. A weighted sum of current and future payoffs can help players to know if some action will not lead into 
lower future stage payoffs. The following discounting payoff function is used to calculate that weighted sum:

$$
\begin{aligned}
\Upsilon_{k}= & (1-\delta) \sum_{t=0}^{\infty} \delta^{t} v_{k}(t), \\
& , \forall j \in\left\{1, \ldots, F_{i}\right\}, \forall i \in\{1, \ldots, G\},
\end{aligned}
$$

for $k=f_{j} \vee g_{i}$, and $0 \leq \delta<1$, which is called discounting factor. With the game progression, $t$ increases and $\delta^{t}$ decreases, meaning that future stage payoffs are less important than current ones. Note that $\delta^{t}$ represents $\delta$ raised to $t$, and not an indexed value. Also, high and low values of $\delta$ characterize players more concerned with future and immediate payoffs, respectively [9].

In each stage $t$, players have to choose their cooperation levels based on strategies. The strategy of a player is a function that indicates the cooperation level to use in every stage $t$. For the forwarding routers, the strategy, $\sigma_{f_{j}}($.$) , depends on$ the downstream and upstream traffic perceived in the previous stage, $\tau_{f_{j}}(t-1), \forall j \in\left\{1, \ldots, F_{i}\right\}, \forall i \in\{1, \ldots, G\}$. Thus:

$$
\lambda_{f_{j}}(t)=\sigma_{f_{j}}\left(\tau_{f_{j}}(t-1)\right) \text {. }
$$

For the gateways, the strategy depends on the cooperation levels of the forwarding routers in the previous stage, $\lambda_{f_{j}}(t-1), \forall j \in\left\{1, \ldots, F_{i}\right\}, \forall i \in\{1, \ldots, G\}$. The gateways treat non forwarding routers as always cooperating forwarding routers. Thus, $\forall j \in\left\{1, \ldots, n_{i}\right\}, \forall i \in\{1, \ldots, G\}$ :

$$
\mu_{r_{j}}^{g_{i}}(t)=\sigma_{g_{i}}\left(\lambda_{r_{j}}(t-1)\right) .
$$

If some forwarding router successfully received and sent a low amount of traffic in the previous stage $(t-1)$, then it can punish other players by forwarding less traffic, i.e. lower $\lambda_{f_{j}}(t), \forall j \in\left\{1, \ldots, F_{i}\right\}, \forall i \in\{1, \ldots, G\}$. Whenever a forwarding router defects, the gateway will be able to punish it by decreasing the respective cooperation level.

\section{Definitions}

Prior to explain simulations and results, we introduce some definitions partly taken from [9]. In the following, we use the term player when referring to either a forwarding router or a gateway, $\sigma_{k}$ represents a strategy of a player $k \in$ $\left\{\mathcal{F}_{i} \cup g_{i}\right\}, \forall i \in\{1, \ldots, G\}$, and $\Sigma_{k}$ represents the set of all the strategies for player $k$.

Definition 1. A strategy profile, $\sigma$, is the set of all the strategies used by all players in some game instance, i.e. $\sigma=\left(\sigma_{f_{1}}, \sigma_{f_{2}}, \ldots, \sigma_{f_{F_{i}}}, \sigma_{g_{i}}\right), \forall i \in\{1, \ldots, G\}$, and $\Sigma$ is the set of all strategy profiles.

Definition 2. $-k$ represents all players except $k$. For instance, the strategy profile $\left(\sigma_{k}, \sigma_{-k}\right)$ includes the strategy $\sigma_{k}$, from player $k$, and the strategy profile from all the other players, $\sigma_{-k}$.

Definition 3. The strategy profile $\sigma=\left(\sigma_{k}, \sigma_{-k}\right)$ is a Nash equilibrium if for all players $k \in\left\{\mathcal{F}_{i} \cup g_{i}\right\}, \forall i \in\{1, \ldots, G\}$ and all strategies $\sigma_{k}^{\prime} \neq \sigma_{k}$,

$$
\Upsilon_{k}(\sigma) \geq \Upsilon_{k}\left(\sigma_{k}^{\prime}, \sigma_{-k}\right),
$$

i.e., there is no other strategy profile that can result in a higher discounted payoff. Here, $\Upsilon_{k}(\sigma)$ refers to the discounting payoff, from (9), when using the strategy profile $\sigma$.

Definition 4. The set of actions played until some stage $t$ is given by the history $h_{t}$. In our model, the actions of the players are the cooperation levels.

Definition 5. The strategy $\sigma_{k}\left(h_{t}\right)$, which is a function dependent on the previous actions, outputs the action that will be used by player $k$ in the next stage $t+1, \forall k=f_{j} \vee g_{i}, \forall j \in$ $\left\{1, \ldots, F_{i}\right\}, \forall i \in\{1, \ldots, G\}$.

Definition 6. $\mathcal{H}_{t}$ is the set of all the possible histories up to time $t$, and $\mathcal{H}$ is the infinite set of all the possible histories for all the possible stages $t$.

Definition 7. Nash equilibrium is borrowed from non repeated game theory and is too permissive in games with nontrivial dynamic structures like the repeated games. Nash equilibrium has no notion of future payoffs, neglecting possible equilibriums beneath a stage not in accordance with Nash equilibrium. The subgame-perfection strengthens Nash equilibrium by taking all the possible histories $\mathcal{H}$ into account. A strategy profile $\sigma$ is a subgame-perfect equilibrium if, for all histories $h_{t} \in \mathcal{H}$, $\sigma\left(h_{t}\right)$ is a Nash equilibrium.

Definition 8. The one-shot deviation allows to check if some strategy profile is subgame-perfect. The principle is to deviate from the strategy at one stage, get back to the strategy in use and check if the deviation leads to a higher payoff. If the deviation leads to a higher payoff then the strategy is not subgame-perfect. Let us fix a strategy profile $\sigma_{-k}$ for the opponents of $k$ and assume a one-shot deviation $\hat{\sigma}_{k}$ from strategy $\sigma_{k}$ of player $k$. The deviation $\hat{\sigma}_{k}$ is profitable if, at some history $h_{t}$, it happens that $h_{t}^{\hat{\sigma}_{k}}=h_{t}^{\sigma_{k}}$ and

$$
\Upsilon_{k}\left(\hat{\sigma}_{k}\left(h_{t+1}\right), \sigma_{-k}\left(h_{t+1}\right)\right)>\Upsilon_{k}\left(\sigma\left(h_{t+1}\right)\right) .
$$

\section{Strategies and Simulation}

\section{A. Strategies}

To study equilibrium in a FiWi access mesh network, we use the following known strategies, that rely on two possible actions: cooperation and non-cooperation [9]. However, the cooperation levels in our game can have any value in $[0,1]$. We consider a low cooperation level as not cooperating, 0.1, and a high cooperation level as cooperating, 0.8.

Tit-For-Tat (TFT): The player mimics the previous action of the opponent. The first action is to cooperate.

Anti-Tit-For-Tat (ATFT): The player mimics the previous action of the opponent. The first action is not to cooperate.

Grim Trigger (GT): Punishes the opponent forever as soon as the opponent does not cooperate.

Always Cooperate (Al-C): Whatever the opponent does, the player always cooperates.

Always Defect (Al-D): Whatever the opponent does, the player never cooperates. 


\section{B. Simulation}

Simulations were done for SFNet in Fig. 1. We consider that nodes receive three times more traffic than they send.

The gain and cost functions, used in (7), are shown to be dependent on $\tau_{f_{j}}(t)$ and $\theta_{f_{j}}(t)$, respectively. To simplify simulations, the gain is assumed to be the amount of traffic successfully sent and received, while the cost is the symmetric of the amount of forwarded traffic. So, the stage payoff of forwarding routers in our simulations is:

$$
\begin{aligned}
v_{f_{j}}^{\prime}(t)= & \tau_{f_{j}}(t)+\left[-\theta_{f_{j}}(t)\right], \\
& \forall j \in\left\{1, \ldots, F_{i}\right\}, \forall i \in\{1, \ldots, G\} .
\end{aligned}
$$

Depending on the network behavior other possibilities exist, e.g. a function that mimics signal interference. The same was considered for the gateway, i.e. the payoff is considered to be the actual amount of traffic that the gateway forwards:

$$
v_{g_{i}}^{\prime}(t)=\theta_{g_{i}}(t), \forall i \in\{1, \ldots, G\} .
$$

In our simulations, the cooperation levels that a gateway $g_{i}$ applies to a router $r_{j}$ is calculated using:

$$
\begin{gathered}
\mu_{r_{j}}^{g_{i}}=\frac{\lambda_{r_{j}}}{\sum_{k=1}^{n_{i}} \lambda_{r_{k}}}, \forall j \in\left\{1, \ldots, n_{i}\right\}, \forall i \in\{1, \ldots, G\} . \\
\text { V. ANALYSIS OF RESULTS }
\end{gathered}
$$

In this section, the one-shot deviation principle was used to search for strategy profiles in equilibrium. Table I shows the strategy profiles in equilibrium with the cooperation levels of 0.1 and 0.8 for defecting and cooperating, respectively, along with the necessary $\delta$ intervals for each player. Notice that in the independent network of gateway 16, when using the oneshot deviation principle, the gateway can make a deviation against node 12 or node 8 . In Table I, only the $\delta$ intervals for the deviation against node 8 are presented since these $\delta$ intervals are smaller and more limitative than the $\delta$ intervals for the deviation against 12 .

Notice that the equilibrium strategies are the same for independent networks of gateways 5, 13 and 22. This happens because these networks have a similar structure: a gateway, a forwarding router, and some non forwarding routers. For the independent network of gateway 16, the set of equilibrium strategy profiles is different, because its structure is different from the previous ones, as shown in Fig. 2.

The $\delta$ intervals for which the strategy profiles are in equilibrium are very large in the independent networks of gateways 5, 13 and 22. Almost all the strategy profiles can have any value for $\delta$, except strategy profile (Al-C, GT). That makes sense because (Al-C, GT) requires the forwarding router to always cooperate no matter what happens in the game, making the deviation more appealing. This means that there are less possible values for $\delta$ to achieve equilibrium. Also, when using (Al-C, GT), the $\delta$ interval required for equilibrium is smaller for the forwarding router at the independent network of gateway 5 . This is due to the fact that more nodes depend on the forwarding router for traffic delivery. For the same strategy profile, (Al-C, GT), the forwarding router 14, from the network of gateway 22, also has a slightly smaller $\delta$ interval, when comparing it with forwarding router 10 , of network 13 , due to the higher number of non forwarding routers dependent on the gateway in that network, as shown in Fig. 2. Hence, having more non forwarding routers dependent on forwarding routers has a high impact on equilibrium.

The $\delta$ interval, besides being used to check how easily a strategy profile can be in equilibrium, can also be used to make a parallel with real network parameters. As explained in Section III-C, high and low values of $\delta$ represent players more concerned with future or current payoffs, respectively. This can be compared with different QoS requirements of services. For instance, VoIP or IPTV are more concerned with immediate payoffs while web browsing and email services are not. As a result, scheduling algorithms can be developed based on strategy profiles for high or low QoS requirements.

In what concerns to the use of strategies by players, we can see that, when in equilibrium, ATFT strategy is used by forwarding routers only. This means that ATFT strategy may be more suitable for forwarding routers. Since this behavior is present for all independent networks, this particularity seems not to be related with the network under study, but more related with different goals of router and gateway players.

Results presented in Table I were obtained using cooperation levels of 0.1 and 0.8 for defecting and cooperating, respectively. However, changing the cooperation level values may change the $\delta$ intervals and can even turn the equilibrium of a strategy profile into non equilibrium. Considering the strategy profile (Al-C, GT) in the independent networks of gateways 5, 13 and 22, Fig. 3(a) and Fig. 3(b) show the increase of the left margin of the $\delta$ interval when the defecting and cooperating cooperation level increase, respectively. Increasing either the cooperating or the defecting cooperation level leads into a narrower $\delta$ interval. This makes sense since higher cooperation levels force forwarding routers to forward more traffic, as can be seen in (5). Deciding for narrow or wide $\delta$ intervals must be investigated when developing scheduling algorithms.

In Fig. 3(c), it is shown that changing cooperation levels can even turn a strategy profile into non-equilibrium. It shows what happens to the left margin of the $\delta$ interval of gateway 16, with strategy profile (TFT, ATFT, TFT), when the cooperation level is too low for cooperating. When it is lower than approximately 0.7 , the left margin of the $\delta$ interval becomes higher than 1 and, as explained in Section III-C, $\delta$ can only have values between 0 and 1 . Notice that, contrarily to the forwarding routers, high cooperating and defecting levels in gateways lead to larger $\delta$ intervals, since their main objective is to forward traffic. This results tell us that it is important to carefully choose the cooperation levels for network players.

\section{CONCLUSIONS AND FUTURE WORK}

In this paper we address the problem of how much bandwidth a wireless router needs to share, for foreign traffic forwarding, so that services of local and foreign users can all receive bandwidth in a balanced way, creating ground for QoS guarantees. A game theoretical approach is proposed. The 


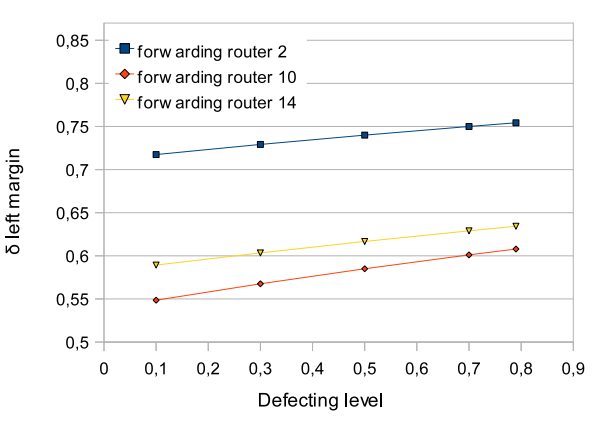

(a)

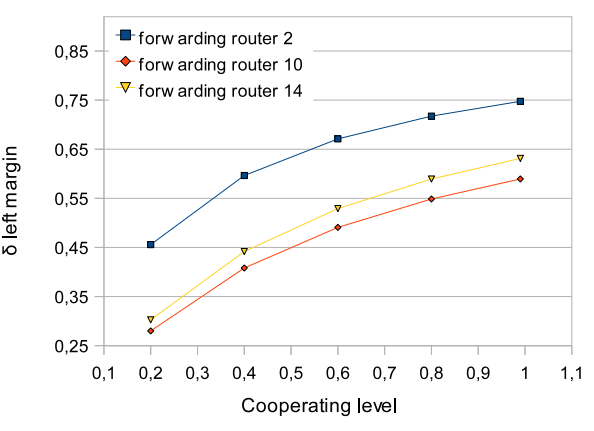

(b)

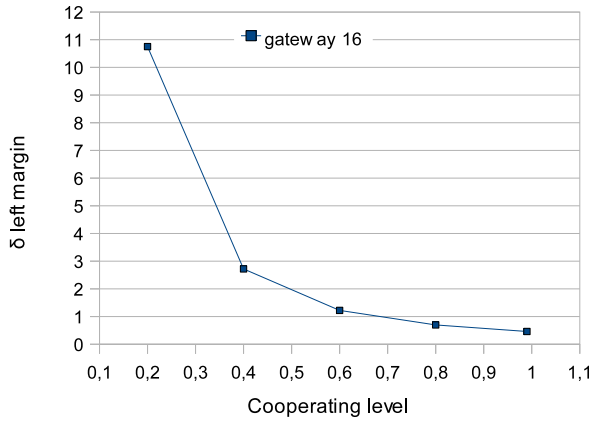

(c)

Fig. 3. $\delta$ interval impacts: a) on forwarding routers when increasing defecting level; b) on forwarding routers when increasing cooperating level c) on gateway 16 when increasing cooperating level.

TABLE I

STRATEGY PROFILES IN EQUILIBRIUM.

\begin{tabular}{|c|c|c|c|c|}
\hline & strategy profiles & $\delta$ inte & als for eq & librium \\
\hline \multirow{8}{*}{$\begin{array}{l}\text { Independent } \\
\text { network of } \\
\text { gateway } \\
16\end{array}$} & $(8,12,16)$ & node 8 & node 12 & $\begin{array}{l}\text { node } 16 \\
\text { deviation } \\
\text { against } 8\end{array}$ \\
\hline & (TFT, ATFT, TFT) & $0.69-1$ & $0-1$ & $0.70-1$ \\
\hline & (ATFT, ATFT, TFT) & $0-0.69$ & $0-1$ & $0.48-1$ \\
\hline & (GT, ATFT, TFT) & $0.69-1$ & $0-1$ & $0.58-1$ \\
\hline & (ATFT, ATFT, GT) & $0-1$ & $0-1$ & $0.48-1$ \\
\hline & (ATFT, ATFT, Al-C) & $0-1$ & $0-1$ & $0.76-1$ \\
\hline & (ATFT, Al-C, GT) & $0-1$ & $0-1$ & $0-1$ \\
\hline & (Al-D, Al-C, GT) & $0-1$ & $0.57-1$ & $0-1$ \\
\hline \multirow{8}{*}{$\begin{array}{l}\text { Independent } \\
\text { network of } \\
\text { gateway } 5\end{array}$} & $(2,5)$ & node 2 & node 5 & \\
\hline & (ATFT, TFT) & $0-1$ & $0-1$ & \\
\hline & (ATFT, GT) & $0-1$ & $0-1$ & \\
\hline & (ATFT, All-C) & $0-1$ & $0-1$ & \\
\hline & $(\mathrm{Al}-\mathrm{C}, \mathrm{GT})$ & $0.72-1$ & $0-1$ & \\
\hline & (Al-D, TFT) & $0-1$ & $0-1$ & \\
\hline & $(\mathrm{Al}-\mathrm{D}, \mathrm{GT})$ & $0-1$ & $0-1$ & \\
\hline & $(\mathrm{Al}-\mathrm{D}, \mathrm{Al}-\mathrm{C})$ & $0-1$ & $0-1$ & \\
\hline \multirow{8}{*}{$\begin{array}{l}\text { Independent } \\
\text { network of } \\
\text { gateway } \\
13\end{array}$} & $(10,13)$ & node 10 & node 13 & \\
\hline & (ATFT, TFT) & $0-1$ & $0-1$ & \\
\hline & (ATFT, GT) & $0-1$ & $0-1$ & \\
\hline & (ATFT, All-C) & $0-1$ & $0-1$ & \\
\hline & (Al-C, GT) & $0.55-1$ & $0-1$ & \\
\hline & (Al-D, TFT) & $0-1$ & $0-1$ & \\
\hline & $(\mathrm{Al}-\mathrm{D}, \mathrm{GT})$ & $0-1$ & $0-1$ & \\
\hline & $(\mathrm{Al}-\mathrm{D}, \mathrm{Al}-\mathrm{C})$ & $0-1$ & $0-1$ & \\
\hline \multirow{8}{*}{$\begin{array}{l}\text { Independent } \\
\text { network of } \\
\text { gateway } \\
22\end{array}$} & $(14,22)$ & node 14 & node 22 & \\
\hline & (ATFT, TFT) & $0-1$ & $0-1$ & \\
\hline & (ATFT, GT) & $0-1$ & $0-1$ & \\
\hline & (ATFT, All-C) & $0-1$ & $0-1$ & \\
\hline & $(\mathrm{Al}-\mathrm{C}, \mathrm{GT})$ & $0.59-1$ & $0-1$ & \\
\hline & (Al-D, TFT) & $0-1$ & $0-1$ & \\
\hline & (Al-D, GT) & $0-1$ & $0-1$ & \\
\hline & (Al-D, Al-C) & $0-1$ & $0-1$ & \\
\hline
\end{tabular}

analysis of results indicate that the equilibrium is possible, depending on the value of $\delta$, which can be translated to a parameter of a scheduling algorithm based on the equilibrium strategy profiles. Also, the ATFT strategy seams to be more suitable to forwarding routers meaning that, in future work, this strategy may be used by algorithms to provide the needed QoS requirements. In future work, the right cooperation levels for cooperation and defection, and their influence when implementing QoS, must be investigated since variations at the $\delta$ interval occur. Technology related case studies must be also analyzed in the future.

\section{ACKNOWLEDGMENT}

This work was supported by FCT (Foundation for Science and Technology) of Portugal within CEOT (Center for Electronic, Optoelectronic and Telecommunications), research project PTDC/EEA-TEL/71678/2006, and by J. Coimbra's Ph.D. grant SFRH/BD/37808/2007.

\section{REFERENCES}

[1] Wei-Tao Shaw, Shing-Wa Wong, Ning Cheng, K. Balasubramanian, Xiaoqing Zhu, M. Maier, L. G. Kazovsky, "Hybrid Architecture and Integrated Routing in a Scalable Optical-Wireless Access Network", IEEE/OSA JLT, vol. 25, no. 11, pp. 3443-3451, Nov. 2007

[2] S. Sarkar, Hong-Hsu Yen, S. Dixit, B. Mukherjee, "Hybrid wirelessoptical broadband access network (WOBAN): network planning and setup", IEEE JSAC, vol. 26, no. 6, pp. 12-21, Aug. 2008

[3] N. Ghazisaidi, M. Maier, C. Assi, "Fiber-wireless (FiWi) access networks: A survey", IEEE Com. Magazine , vol. 47, no. 2, pp. 160-167, Feb. 2009

[4] S. Sarkar, S. Dixit, B. Mukherjee, "Hybrid Wireless-Optical BroadbandAccess Network (WOBAN): A Review of Relevant Challenges", IEEE/OSA JLT, vol. 25, no. 11, pp. 3329-3340, Nov. 2007

[5] M. Félegyházi, J. P. Hubaux, L. Buttyan, "Nash equilibria of packet forwarding strategies in wireless ad hoc networks", IEEE Transactions on Mobile Computing, vol. 5, no. 5, pp. 463-476, May 2006

[6] M. Félegyházi, "Non-Cooperative Behavior in Wireless Networks", Ph.D. dissertation, École Polytechnique Fédérale de Lausanne, 2007

[7] V. Srivastava, J. Neel, A. B. Mackenzie, R. Menon, L. A. Dasilva, J. E. Hicks, J. H. Reed, R. P. Gilles, "Using game theory to analyze wireless ad hoc networks", IEEE Com. Surveys \& Tutorials, vol. 7, no. 4, pp. 46-56, Fourth Quarter 2005

[8] M. Felegyhazi, M. Cagalj, S. S. Bidokhti, J. P. Hubaux, "Non-Cooperative Multi-Radio Channel Allocation in Wireless Networks", IEEE INFOCOM 2007, pp. 1442-1450, May 2007

[9] George J. Mailath and L. Samuelson, Repeated Games and Reputations: Long-Run Relationships, Oxford University Press, USA, 2006. 